\title{
Universal Fermi Gas with Two- and Three-Body Resonances
}

\author{
Yusuke Nishida, Dam Thanh Son, and Shina Tan \\ Institute for Nuclear Theory, University of Washington, Seattle, Washington 98195-1550, USA
}

(Dated: February 27, 2008)

\begin{abstract}
We consider a Fermi gas with two components of different masses, with the $s$-wave two-body interaction tuned to unitarity. In the range of mass ratio $8.62<M / m<13.6$, it is possible for a short-range interaction between heavy fermions to produce a resonance in a three-body channel. The resulting system is scale invariant and has universal properties, and is very strongly interacting. When $M / m$ is slightly above the lower limit 8.62 , the ground state energy of a 2:1 mixture of heavy and light fermions is less than $2 \%$ of the energy of a noninteracting gas with the same number densities. We derive exact relationships between the pressures of the unitary Fermi gases with and without three-body resonance when the mass ratio is close to the critical values of 8.62 and 13.6. Possible experimental realization with cold atoms in optical lattices is discussed.
\end{abstract}

PACS numbers: 05.30.Fk, 03.75.Ss

Introduction.- Dilute Fermi gas at infinite scattering length $[1-3]$ has attracted considerable attention recently. The system can be realized in atomic traps using the Feshbach resonance [4-9]. This system is called Fermi gas at unitarity and has interesting universal properties. For example, the energy of the ground state of a symmetric Fermi gas at unitarity is $E=\xi E_{\text {free }}$, where $E_{\text {free }}$ is the energy of a noninteracting system with the same density and $\xi$ is a dimensionless number. Most recent evaluations for $\xi$ yield $\xi \approx 0.4$.

Other systems with universal properties that have been considered so far are variations of this unitary Fermi gas. One can consider different densities of the two fermion components $[10,11]$, or different masses of the two fermions, or both $[12,13]$. Here we show that there exists a novel type of system with both two- and threebody interactions fine-tuned to resonance.

Three-body wave function.- - In order to realize a threebody resonance, it is necessary to allow the two components of the Fermi gas to have different masses. We shall denote the mass of the heavy fermion as $M$ and of the light fermion as $m$. The ratio $u=M / m$ is a free parameter.

Let us recall what is known for three-body physics, using the Born-Oppenheimer approximation at first [14], but then proceed to the exact result. Consider a threebody system of two heavy and one light fermions. If $M \gg$ $m$ one can use the adiabatic approximation, separating the wave function into the fast part of the light fermion and the slow part of the heavy fermions,

$$
\psi\left(\boldsymbol{x}_{1}, \boldsymbol{x}_{2}, \boldsymbol{y}\right)=\psi_{\text {slow }}\left(\boldsymbol{x}_{1}, \boldsymbol{x}_{2}\right) \psi_{\text {fast }}\left(\boldsymbol{y} ; \boldsymbol{x}_{1}, \boldsymbol{x}_{2}\right) .
$$

The function $\psi_{\text {fast }}\left(\boldsymbol{y} ; \boldsymbol{x}_{1}, \boldsymbol{x}_{2}\right)$ satisfies the Schrödinger equation of one particle moving in the field of two static centers:

$$
-\frac{\hbar^{2}}{2 m} \nabla_{\boldsymbol{y}}^{2} \psi_{\text {fast }}\left(\boldsymbol{y} ; \boldsymbol{x}_{1}, \boldsymbol{x}_{2}\right)=E\left(\boldsymbol{x}_{1}, \boldsymbol{x}_{2}\right) \psi_{\text {fast }}(\boldsymbol{y}) .
$$

The resonant two-body interaction implies that the asymptotics of $\psi_{\text {fast }}(\boldsymbol{y})$ when $\boldsymbol{y} \rightarrow \boldsymbol{x}_{i}, i=1,2$ is
$\left|\boldsymbol{y}-\boldsymbol{x}_{i}\right|^{-1}+O\left(\left|\boldsymbol{y}-\boldsymbol{x}_{i}\right|\right)$. One finds one negative energy level with

$$
E(R)=-\frac{C^{2} \hbar^{2}}{2 m R^{2}}, \quad R=\left|\boldsymbol{x}_{1}-\boldsymbol{x}_{2}\right|,
$$

where $C=0.5671 \ldots$ is the solution to equation $e^{-x}=x$. We then regard $E(R)$ as the potential energy between the two heavy fermions. Since it is a $R^{-2}$ potential, the wave function $\psi_{\text {slow }}$ near the origin behaves as $R^{\alpha} Y_{l m}(\theta, \phi)$, where $\alpha$ is a solution to the equation

$$
\alpha(\alpha+1)=l(l+1)-\frac{C^{2} u}{2} .
$$

Because of the Fermi statistics $l$ is odd. When the righthand side of Eq. (4) is greater than $-\frac{1}{4}$, there are two real solutions $\alpha_{+}$and $\alpha_{-}$, relating to each other by $\alpha_{+}+\alpha_{-}=$ $-1\left(\alpha_{+}>-1 / 2>\alpha_{-}\right)$. If the right-hand side is less than $-\frac{1}{4}$, which is the case for $l=1$ and $u>u_{\max } \approx 14.0$, then $\alpha$ is complex. This corresponds to the Efimov effect, where the system develops deep bound states in the zerorange limit, which break scale invariance.

In the former case, $u<u_{\max }$, there are two further possibilities. If $\alpha_{+}>\frac{1}{2}$ and $\alpha_{-}<-\frac{3}{2}$, then the solution $\psi_{\text {slow }} \sim R^{\alpha_{-}}$is not normalizable at the origin. Therefore, the only consistent boundary condition for $\psi_{\text {slow }}$ at the origin is $\psi_{\text {slow }} \sim R^{\alpha_{+}}$. However, when $\alpha_{+}<\frac{1}{2}$, $\alpha_{-}>-\frac{3}{2}$, both asymptotics $R^{\alpha_{ \pm}}$are normalizable at the origin. Therefore, generically $\psi_{\text {slow }}$ will behave as

$$
\psi_{\text {slow }}(R, \theta, \phi)=\left(c_{+} R^{\alpha_{+}}+c_{-} R^{\alpha_{-}}\right) Y_{l m}(\theta, \phi),
$$

where the ratio $c_{+} / c_{-}$is determined by the interaction. For a generic interaction $c_{+} / c_{-}$, which has dimension [length] ${ }^{\alpha_{-}-\alpha_{+}}$, will be of order $r_{0}^{\alpha_{-} \alpha_{+}}$, where $r_{0}$ is the characteristic interaction range. That means that at distances $R \gg r_{0}$ the wave function behaves like $R^{\alpha_{+}}$. However, by including an attractive force between the heavy fermions (in addition to the effective attraction induced by the light fermion), and fine-tuning its strength, one can achieve the situation where only the coefficient of the 
$R^{\alpha_{-}}$piece is very large. This corresponds to a three-body resonance, and $c_{+}=0$ corresponds to this resonance being at threshold. For $l=1$, this is possible when $u>$ $u_{\min } \approx 7.77$. To verify our point, we considered a model potential $V(R)=\left(\hbar^{2} / M\right)\left(\beta_{1} / R^{12}-\beta_{2} / R^{6}\right)$ between the heavy fermions. Within the Born-Oppenheimer approximation, when $\beta_{2} / \beta_{1}^{2 / 5} \approx 6.5+2.9 \alpha_{+}\left(-\frac{1}{2} \leq \alpha_{+} \leq\right.$ $\frac{1}{2}$ ), which is not a strong enough attraction to produce a two-body resonance, the desired three-body resonance is achieved.

The Born-Oppenheimer approximation employed above is not entirely justified, because the obtained values of $u_{\min }$ and $u_{\max }$ are not parametrically large. The conclusions, however, are confirmed by an exact treatment of the three-body problem. The wave function $\Psi\left(\boldsymbol{x}_{1}, \boldsymbol{x}_{2}, \boldsymbol{y}\right)$ satisfies the free Schrödinger equation with the two-body boundary condition when $\left|\boldsymbol{x}_{i}-\boldsymbol{y}\right| \rightarrow 0$. In hyperspherical coordinates, its behavior is

$$
\Psi=R^{\gamma} f_{l}(\Omega),
$$

where $R$ is the hyperradius and $\Omega$ denotes the hyperangular variables, and $\gamma$ is related to $\alpha$ by $\gamma=\alpha-\frac{3}{2}$. For the $l=1$ channel, the power $\gamma$ is determined from the equation [15]

$$
\begin{array}{r}
\frac{\cos \left[(\gamma+1) \arccos \frac{u}{u+1}\right]-\cos \left[(\gamma+1) \arccos \frac{-u}{u+1}\right]}{(\gamma+1) \sin \pi \gamma} \\
+\frac{\cos \left[(\gamma+3) \arccos \frac{u}{u+1}\right]-\cos \left[(\gamma+3) \arccos \frac{-u}{u+1}\right]}{(\gamma+3) \sin \pi \gamma} \\
=-\frac{2 u^{2}}{(u+1)^{3}} \sqrt{2 u+1} .
\end{array}
$$

Solving this equation, we find that $u_{\max } \approx 13.6$ and $u_{\min } \approx 8.62$, which somewhat differ from the values obtained in the adiabatic approximation. At the qualitative level, however, the physics is the same: for the mass ratio between these two values, one can fine-tune one three-body channel to resonance. Note that the mass ratio $u_{\text {min }} \approx 8.62$ played a special role in the discussion of three-body recombination in Ref. 15.

Many-body physics. - We now discuss the ground state energy of the many-body system with both two- and three-body resonances. The first question is whether the system is stable toward collapse. Such a collapse can happen if there exists an Efimov state of four or more particles for the mass ratio between 8.62 and 13.6. This would be very unusual, as it is before the three-body Efimov state appears. Consider four-body states. In the BornOppenheimer approximation, there is no Efimov state of two heavy and two light fermions, since there is only one bound state in the Schrödinger equation with two static centers. The case of three heavy and one light fermion is more subtle, but here the three centrifugal potentials between the heavy fermions make the Efimov effect less likely. We shall proceed under the assumption that there is no Efimov effect for any number of heavy and light particles, and the system is stable.

Let us define $\xi$ as the ratio of the ground state energy $E$ to the ground state energy $E_{\text {free }}$ of the noninteracting system at the same densities of the heavy and the light components, $n_{h}$ and $n_{l}$, respectively,

$$
E\left(n_{h}, n_{l}\right)=\xi E_{\text {free }}\left(n_{h}, n_{l}\right) .
$$

Since the system has no intrinsic dimensionful parameter, $\xi$ can depend only on two variables: the mass ratio $M / m$, and the relative abundance of the two components. For the latter, we define

$$
x=\frac{n_{l}}{n_{h}+n_{l}} .
$$

In general, the problem of finding $\xi(M / m, x)$ is difficult, since there is no small parameter in the problem. We shall concentrate on the case when $M / m$ is slightly larger than the lower critical value 8.62. In this case, the normalization integral for the three-body wave function is dominated at small distances. Thus, one can think about the three-body system as a localized bound state. The situation is analogous to the behavior of the twobody state at resonance near four spatial dimensions. As the number of spatial dimensions $d$ approaches four from below, the two-body wave function becomes more and more dominated by short distances, which has led Nussinov and Nussinov to suggest that $\xi \rightarrow 0$ in this limit [16]. This was confirmed by the $\epsilon$ expansion near $d=4$ [17].

The view that the three-body system behaves as a tightly bound state is confirmed by its behavior in an isotropic harmonic potential. It is known that each exponent $\gamma$, determined from solving the free-space Schrödinger equation, is related to an energy level of the three particles in an isotropic harmonic potential [18],

$$
E_{0}=\left(\frac{9}{2}+\gamma\right) \hbar \omega
$$

where $\omega$ is the oscillator frequency. When $\gamma \rightarrow-3$, the energy approaches $\frac{3}{2} \hbar \omega$. This is consistent with the picture of the trimer being a localized bound state of all three particles. We emphasize that the three-body state is not a real bound state with a finite binding energy. In particular, in the harmonic trap there are breathing modes, corresponding to a ladder of energy excitations $E_{0}+2 n \hbar \omega, n=1,2, \ldots[19]$, that are absent for the single particle. However, when the trimer is in the ground state with respect to the breathing mode, it behaves as a pointlike particle.

Having in mind this picture of trimers as objects localized in space, let us put an upper bound on the value of $\xi$ at $x=\frac{1}{3}$, and $u=8.62+\epsilon$. In this case one can combine all heavy and light particles (whose relative number is 2:1) into trimers, and construct a dilute gas of such trimers. This gas will be a weakly interacting Fermi gas 
of trimers with three possible polarizations (the trimer has $l=1$ ). Its energy density is therefore

$$
\frac{E_{\text {trimer }}}{V}=c \frac{n_{l}^{5 / 3}}{3^{2 / 3}(2 M+m)},
$$

where $c=\frac{3}{10}\left(6 \pi^{2}\right)^{2 / 3} \hbar^{2}$ and $n_{l}$ is the density of the light fermions. On the other hand, if one turns off the interaction, the system becomes a Fermi gas of light fermions with density $n_{l}$ plus a Fermi gas of heavy fermions with density $n_{h}=2 n_{l}$. The energy density of this gas is

$$
\frac{E_{\text {free }}}{V}=c \frac{n_{l}^{5 / 3}}{m}+c \frac{\left(2 n_{l}\right)^{5 / 3}}{M} .
$$

Taking the ratio we find

$$
\frac{E_{\text {trimer }}}{E_{\text {free }}}=\frac{u}{3^{2 / 3}\left(u+2^{5 / 3}\right)(2 u+1)} \approx 1.93 \times 10^{-2} .
$$

It is clear that the trimer gas energy is not the lowest ground state energy and hence is only an upper bound on the latter. For example, a trimer with energy near the Fermi energy can decay into its constituents (two heavy and one light fermions) since the binding energy is zero, and this decay can happen until chemical equilibrium is reached. The true ground state is therefore a mixture of the trimer gas with a gas of constituent fermions. Because of the strong interactions between constituent fermions, we do not have an exact formula for the ground state energy. The upper bound, on the other hand, implies that the system is very strongly interacting: the ground state energy is less than $2 \%$ of the energy of the noninteracting system: $\xi\left(u \rightarrow u_{\min }, \frac{1}{3}\right)<1.93 \times 10^{-2}$. This can be contrasted with the case of symmetric unitary Fermi gas with equal masses, where $\xi \approx 0.4$.

For a general value of $x$, we can put an upper bound on $\xi$ by considering the state where the maximal number of trimers is bound and form a trimer Fermi gas, while the remainder (heavy fermions for $x<\frac{1}{3}$ and light fermions for $x>\frac{1}{3}$ ) form another free Fermi gas. The resulting bound is plotted in Fig. 1. One sees that the variational bound is lowest when $x$ is close to $1 / 3$. From the point of view of the ground state energy, the 2:1 mixture of heavy and light fermions is therefore the most interesting.

Exact relationships near $u=8.62$ and 13.6. - For mass ratios $u$ between 8.62 and 13.6, therefore, there exist two scale invariant regimes, which give rise to unitary Fermi gases with and without three-body resonance. We now show that near the lower critical value $u_{\min }$ there exists an exact relationship between the pressures of a unitary Fermi gas with three-body resonance, $P$, and a unitary Fermi gas without three-body resonance, $P_{0}$, at the same chemical potentials of light and heavy fermions, $\mu_{h}$ and $\mu_{l}$. Namely,

$$
\begin{aligned}
\lim _{u \rightarrow u_{\min }}\left[P\left(\mu_{l}, \mu_{h}\right)\right. & \left.-P_{0}\left(\mu_{l}, \mu_{h}\right)\right] \\
= & \frac{[2(2 M+m)]^{3 / 2}}{5 \pi^{2} \hbar^{3}}\left(2 \mu_{h}+\mu_{l}\right)^{5 / 2} .
\end{aligned}
$$

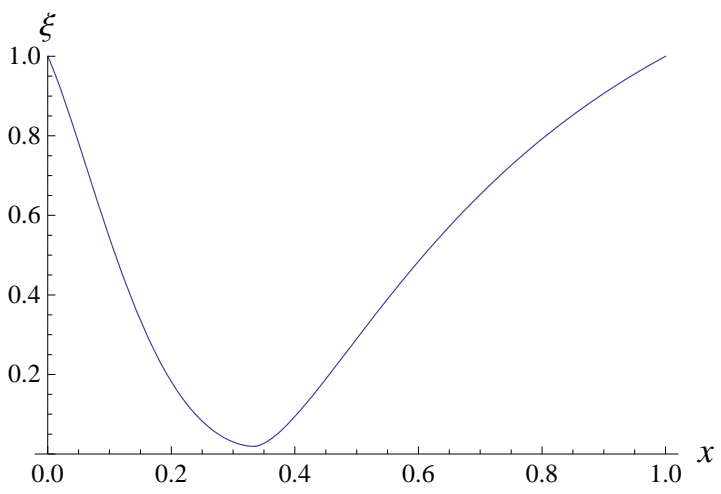

FIG. 1: The variational bound on $\xi$ as a function of the abundance of light fermions $x$ at $M / m=8.62+\epsilon$.

We emphasize here that we have in mind the limit when for each mass ratio $u$ we take the zero-range limit, compute the pressures $P$ and $P_{0}$, and then take the limit $u \rightarrow u_{\text {min }}$. In general the limit $u \rightarrow u_{\min }$ and the zerorange limit do not commute.

The right-hand side of Eq. (14) is the pressure of an ideal Fermi gas of trimers, with $l=1$, at chemical potential $2 \mu_{h}+\mu_{l}$. Physically, Eq. (14) means that at $u \rightarrow u_{\text {min }}$ the unitary Fermi gas with three-body resonance consists of two parts: a trimer gas and a Fermi gas of the fermionic constituents outside the trimers. These two parts interact weakly with each other, but are in chemical equilibrium.

When $x=\frac{1}{3}$, there is another simple relationship between the ground state energies with and without threebody resonance. $\xi$ of the Fermi gas with three-body resonance is determined by the analogously defined quantity of the unitary Fermi gas without three-body resonance $\xi_{0}$ via

$$
\xi^{-3 / 2}=\xi_{0}^{-3 / 2}+374 . \quad\left(x=\frac{1}{3}, u \rightarrow u_{\min }\right) .
$$

We have argued above that the trimers decouple at $u_{\text {min }}$. What is less trivial is that the fermions outside the trimers contribute the same pressure as a Fermi gas without three-body resonance. This seems strange: the three-body wave functions satisfy different boundary conditions at small distances $\left(R^{\gamma_{-}}\right.$vs. $\left.R^{\gamma_{+}}\right)$in the two cases. To see that it is the case, consider the three-body wave function of three particles at some positive energy. The radial part of the Schrödinger equation reads

$$
\frac{\partial^{2} \psi}{\partial R^{2}}+\frac{5}{R} \frac{\partial \psi}{\partial R}-\frac{\gamma(\gamma+4)}{R^{2}} \psi=-k^{2} \psi(R)
$$

This equation has two solutions:

$$
\psi(R) \sim R^{-2} J_{ \pm \nu}(k R), \quad \nu=|\gamma+2|,
$$

where $J_{-\nu}$ corresponds to the case with three-body resonance, and $J_{\nu}$ to that without the resonance. The limit 


$$
\begin{array}{c|c|c|c|c|c|c|c}
t_{l} / t_{h} & 8.62 & 9 & 10 & 11 & 12 & 13 & 13.6 \\
\hline \multirow{2}{*}{W / t_{h}} & -3.80 & -3.625 & -3.15 & -2.575 & -2.0 & -1.2 & >-0.4 \\
& \pm 0.03 & \pm 0.025 & \pm 0.05 & \pm 0.075 & \pm 0.1 & \pm 0.1 &
\end{array}
$$

TABLE I: Approximate values of $W / t_{h}$ for three-body resonance on a lattice.

$u \rightarrow u_{\text {min }}$ corresponds to $\nu \rightarrow 1$. By using the relationship between the Bessel functions,

$$
J_{\nu}(z) \cos (\nu \pi)-J_{-\nu}(z)=\sin (\nu \pi) Y_{\nu}(z),
$$

one sees that in the limit $\nu \rightarrow 1$ the two wave functions in Eq. (17) are the same up to a sign, except for a very small region near the origin, $k R \sim \sqrt{1-\nu}$. This means that, for the fermions outside the trimers, which have finite kinetic energy, the resonant and nonresonant boundary conditions are equivalent. This leads to the relationships (14) and (15).

On the other hand, the limit $u \rightarrow u_{\max }$ corresponds to $\nu \rightarrow 0$, where two solutions in Eq. (17) coincide. Therefore there is no distinction between Fermi gases with and without three-body resonance and we obtain another exact relationship

$$
\lim _{u \rightarrow u_{\max }}\left[P\left(\mu_{l}, \mu_{h}\right)-P_{0}\left(\mu_{l}, \mu_{h}\right)\right]=0 .
$$

Other channels. - So far we have considered only the $l=1$ three-body channel. One may ask whether similar fine-tuning can be done in other channels. To answer this question, one has to compute the exponents $\gamma_{+}$in other channels and look for the mass ratio $u$ where it crosses -1 . This crossing happens only for odd $l$. For $l=3$ it occurs at $u=70.1$, and the value of the mass ratio increases with increasing $l$. For these large mass ratios, the Efimov effect has already taken hold in the $l=1$ channel, and the system cannot be scale-invariant. We conclude that scale invariance with resonant three-body interaction can be achieved only in the $l=1$ channel.

Experimental realization in a lattice.- Fermions of species $c$ and $b$ on a cubic lattice with the Hamiltonian

$$
\begin{aligned}
H=-\sum_{<i j>}\left(t_{h} c_{i}^{\dagger} c_{j}+t_{l} b_{i}^{\dagger} b_{j}\right) & +U \sum_{i} c_{i}^{\dagger} b_{i}^{\dagger} b_{i} c_{i} \\
& +\frac{1}{2} W \sum_{<i j>} c_{i}^{\dagger} c_{j}^{\dagger} c_{j} c_{i}
\end{aligned}
$$

(where $\sum_{\langle i j\rangle}$ extends over nearest-neighbor lattice sites) approach the above universal Fermi gas limit when the average number of fermions per lattice site $\ll 1$, if parameters are tuned to get $c b$ and $c c b$ resonances: $U /\left(t_{h}+t_{l}\right) \approx$ $-3.957,8.62 \leq t_{l} / t_{h} \leq 13.6$, and numerically determined $W$ is shown in Tab. I (currently we only know a lower bound for $W$ at $\left.t_{l} / t_{h}=13.6\right)$. One can easily show that $p$-wave $c c$ resonance is not reached until $W / t_{h} \approx-9.53$.

The model (20) may be experimentally realized with cold atoms on an optical lattice. If the $c b$ scattering length in free space is small and negative, we can tune $c b$ to resonance by tuning the lattice depth [20]. $t_{l} / t_{h}$ is also tunable. Nearest-neighbor attraction between $c$ atoms may be mediated by a third kind of atom having a much smaller effective mass than $c$ and $b$, interacting with $c$ atoms with an effective scattering length $a_{c 3}: a_{c 3}<0$ and $\left|a_{c 3}\right|$ slightly exceeds the lattice spacing. Tuning $a_{c 3}$ with a magnetic field near a $c 3$ Feshbach resonance, we can tune the magnitude of $W$ in Eq. (20). The lattice also dramatically increases the lifetime of the system by suppressing the three-body recombination rate [21].

Conclusion.- We have argued that there exists a class of universal Fermi gases with resonances in both twoand three-body channels. This system remains strongly coupled in the whole range of mass ratio, $8.62<M / m<$ 13.6, where it exists. We have discussed the possibility of realizing these gases with cold atoms in an optical lattice. The relevant lattice model, Eq. (20), may also be studied with quantum Monte-Carlo simulations.

We thank Soon Yong Chang for discussions. Y.N. is supported, in part, by JSPS Postdoctoral Program for Research Abroad. This work is supported, in part, by DOE Grant No. DE-FG02-00ER41132.

[1] D. M. Eagles, Phys. Rev. 186, 456 (1969).

[2] A. J. Leggett, in Modern Trends in the Theory of Condensed Matter (Springer, Berlin, 1980).

[3] P. Nozières and S. Schmitt-Rink, J. Low Temp. Phys. 59, 195 (1985).

[4] K. M. O'Hara et al., Science 298, 2179 (2002).

[5] C. A. Regal, M. Greiner, and D. S. Jin, Phys. Rev. Lett. 92, 040403 (2004).

[6] M. Bartenstein et al., Phys. Rev. Lett. 92, 120401 (2004).

[7] M. W. Zwierlein et al., Phys. Rev. Lett. 92, 120403 (2004).

[8] J. Kinast et al., Phys. Rev. Lett. 92, 150402 (2004).

[9] T. Bourdel et al., Phys. Rev. Lett. 93, 050401 (2004).

[10] M. W. Zwierlein et al., Science 311, 492 (2006).

[11] G. B. Partridge et al., Science 311, 503 (2006).

[12] M. Iskin and C. A. R. Sa de Melo, Phys. Rev. Lett. 97, 100404 (2006).

[13] S.-T. Wu, C.-H. Pao, and S.-K. Yip, Phys. Rev. B 74, 224504 (2006).

[14] A. C. Fonseca, E. F. Redish, and P. E. Shanley, Nucl. Phys. A320, 273 (1979).

[15] D. S. Petrov, Phys. Rev. A 67, 010703(R) (2003).

[16] Z. Nussinov and S. Nussinov, Phys. Rev. A 74, 053622 (2006).

[17] Y. Nishida and D. T. Son, Phys. Rev. Lett. 97, 050403 (2006).

[18] S. Tan, cond-mat/0412764.

[19] F. Werner and Y. Castin, Phys. Rev. Lett. 97, 150401 (2006); Phys. Rev. A 74, 053604 (2006).

[20] P. O. Fedichev, M. J. Bijlsma, and P. Zoller, Phys. Rev. Lett. 92, 080401 (2004).

[21] B. Marcelis, S. J. J. M. F. Kokkelmans, G. V. Shlyapnikov, and D. S. Petrov, arXiv:0711.4632v1. 